\title{
Long-term prognostic significance of the ST level and ST slope in the 12-lead ECG in the general population
}

\author{
Istolahti, Tiia
}

2020

Istolahti , T , Nieminen , T , Huhtala , H , Lyytikäinen , L-P , Kähönen , M , Lehtimäki , T, Eskola , M , Anttila , I , Jula , A , Rissanen , H , Nikus , K \& Hernesniemi , J 2020 , ' Long-term prognostic significance of the ST level and ST slope in the 12-lead ECG in the general population ' , Journal of Electrocardiology , vol. 58 , pp. 176-183 . https://doi.org/10.1016/j.jelectrocard.2019

http://hdl.handle.net/10138/327216

https://doi.org/10.1016/j.jelectrocard.2019.12.010

publishedVersion

Downloaded from Helda, University of Helsinki institutional repository.

This is an electronic reprint of the original article.

This reprint may differ from the original in pagination and typographic detail.

Please cite the original version. 


\title{
Long-term prognostic significance of the ST level and ST slope in the 12-lead ECG in the general population
}

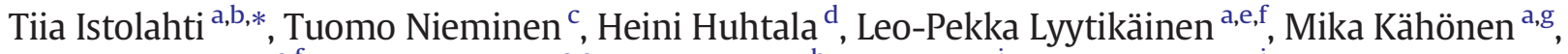 \\ Terho Lehtimäki ${ }^{\text {a,f }}$, Markku Eskola a,e, Ismo Anttila ${ }^{\text {h }}$, Antti Jula ${ }^{\mathrm{i}}$, Harri Rissanen ${ }^{\mathrm{i}}$, \\ Kjell Nikus ${ }^{\text {a,e }}$, Jussi Hernesniemi ${ }^{\text {a,e }}$
}

a Faculty of Medicine and Health Technology, Tampere University, and Finnish Cardiovascular Research Center, Tampere, Finland

b Department of Internal Medicine, Vaasa Central Hospital, Vaasa, Finland

c Department of Internal Medicine, Päijät-Häme Central Hospital, Lahti, Finland

d Faculty of Social Sciences, Tampere University, Tampere, Finland

e Heart Center, Department of Cardiology, Tampere University Hospital, Tampere, Finland

${ }^{\mathrm{f}}$ Department of Clinical Chemistry, Fimlab Laboratories, Tampere, Finland

${ }^{g}$ Department of Clinical Physiology, Tampere University Hospital, Tampere, Finland

${ }^{\text {h }}$ Department of Emergency Services, Seinäjoki Central Hospital, Seinäjoki, Finland

${ }^{\mathrm{i}}$ National Institute for Health and Welfare, Helsinki, Finland

\section{A R T I C L E I N F O}

\section{Keywords:}

ST level

ST slope

Population study

Outcome

ECG

\begin{abstract}
A B S T R A C T
Background: Even minor ST depression in the electrocardiogram (ECG) is associated with cardiovascular disease and increased mortality. There is limited data on the prognostic significance of ST-level changes in the general population.

Subjects and methods: A random sample of Finnish subjects $(\mathrm{n}=6354)$ aged over 30 years $(56.1 \%$ women $)$ underwent a health examination including a 12-lead ECG in the Health 2000 survey. The effects of relative ST level as a continuous variable and ST slope (upsloping, horizontal, downsloping) in three different lead groups were analyzed using a multi-adjusted Cox proportional hazard model separately for men and women with total mortality as endpoint.

Results: The follow-up lasted for 13.7 (SD 3.3) years for men and 13.9 (SD 3.1) years for women. Lower lateral ST levels were associated with all-cause mortality in multi-adjusted models in both genders (at $\mathrm{J}+80 \mathrm{~ms}$ hazard ratio [HR] 0.64 for a change of $1.0 \mathrm{~mm}$ [95\% confidence interval $0.49-0.84, \mathrm{p}=0.002$ ] for men and HR 0.61 [0.48-0.78, $\mathrm{p}<0.001]$ for women). Associated coronary heart disease had no major influence on the results. Exclusion of subjects with ECG signs of left ventricular hypertrophy from the analyses increased the mortality risk of lower lateral ST levels in men but decreased it in women. For the anterior and inferior lead groups, no statistically significant difference was seen after multivariate adjustment. ST slope was not an independent predictor of mortality after multivariate adjustment.

Conclusion: Lower ST level in the lateral ECG leads is an independent prognostic factor to predict all-cause mortality in the general population.
\end{abstract}

(c) 2019 Elsevier Inc. All rights reserved.

\section{Introduction}

Abnormalities in the electrocardiographic (ECG) ST level reflect abnormalities in ventricular repolarization. In earlier population studies, major ST depression has been associated with worse outcome and risk for cardiovascular disease [1-3], and also minor ST changes had an adverse effect on prognosis [4-7]. In most of the studies, outcome was

\footnotetext{
* Corresponding author at: Department of Internal Medicine, Vaasa Central Hospital, Hietalahdenkatu 2-4, 65100 Vaasa, Finland.

E-mail address: tiia.istolahti@tuni.fi (T. Istolahti).
}

determined by the magnitude of these ECG changes [4]. Some studies even suggested that ST depression was associated cardiovascular death in a "dose-response" manner [8]. However, there are only few studies examining the ST level as a continuous instead of a categorical variable.

A downsloping ST segment, especially in exercise testing, is considered typical for coronary artery disease [9]. Also, a downward sloping ST segment together with T-wave inversion in the lateral leads, the "strain pattern", is a marker of anatomical left ventricular hypertrophy (LVH) and is associated with increased left ventricular mass and mortality [10]. There is very limited data on the prognostic value of ST slope as 
a separate ECG parameter (independently of the ST level), especially in the general population.

The prognostic value of ST changes is dependent of the location of the changes within the 12-lead ECG $[8,11]$. Nevertheless, many authors did not specify the location of ST changes in their analyses. Gender differences in cardiovascular diseases have received attention in preventive cardiology. Previously most of the studies dealing with ST changes included more men than women. Lately, some studies have also focused on women, and it seems that the prognostic significance of these ECG changes is similar in both genders, especially when it comes to minor changes $[3,4,12,13]$.

The aim of this study was to establish the prognostic role of the level of the ST segment and of the ST slope, separately in three different anatomical lead groups, in both men and women in the general population.

\section{Materials and methods}

\section{Study population}

The study is based on the Health 2000 survey, which was carried out in 2000-2001. The study design has been described more in detail previously [14]. The population-based nationwide study consisted of 8028 individuals aged over 30 years, of whom 79\% (6354 individuals) participated in the health examination, which included a structured examination by a physician, series of laboratory tests and an ECG recording. The study was designed to cover a nationally representative population sample of the Finnish population. Individuals aged $80+$ were oversampled using double inclusion probabilities. Ethical approval for the Health 2000 study was obtained from the Ethical Committee for Research in Epidemiology and Public Health at the Hospital District of Helsinki and Uusimaa (HUS).

\section{ECG registration, definitions and analysis}

During the health examination, a standard 12-lead resting ECG in supine position was recorded from each subject with Marquette Hellige MAC 5000 electrocardiographs (Freiburg, Germany and Milwaukee, WI, USA). ECGs were stored electronically and printed at a paper speed of $50 \mathrm{~mm} / \mathrm{s}$. The low-pass filter was set at $150 \mathrm{~Hz}$. If the participant had significant muscular tremors that interfered with the recording, the filter was set at $40 \mathrm{~Hz}$. High-pass filter $0.16 \mathrm{~Hz}$ was used. The Marquette 12SL program can use high-pass filter at $0.32 \mathrm{~Hz}$ without inducing artifacts to the ST level measurements. The ECG data were sent for further analysis to the Social Insurance Institution's research center in Turku, where ECGs were analyzed with Magellan software (Marquette Electronics Inc., Milwaukee, WI, USA). ST levels and durations and amplitudes of different waves of every single 12-lead ECG were measured. The Marquette 12SL algorithm uses median complexes of the 10-s ECG tracing and uses the onset of QRS as an isoelectric line. The Minnesota coding was performed at the Institute of Cardiology, Kaunas Medical Academy, Lithuania, by two investigators blinded to the clinical data. The repeatability of the Minnesota Code was checked by repeat analysis of 200 ECGs.

The following three lead groups were used in the present study: anterior (leads V1-V4), lateral (aVL, I, V5-V6) and inferior (II, aVF, III). In addition, lead V5 was included as a single lead. ST levels were measured at the J point and $80 \mathrm{~ms}$ after the J point $(\mathrm{J}+80 \mathrm{~ms})$. The lead with the lowest ST level in a particular lead group was used for analysis. An additional parameter "Jalt $\mathrm{J}+80 \mathrm{~ms}$ " was also created, and it was defined as the lower ST level of the J point and J +80 ms measurement points. ST slope was defined as the difference between the ST level at the $\mathrm{J}+80 \mathrm{~ms}$ and $\mathrm{J}$ point in a single lead. If the absolute value of difference between the measurement point at $\mathrm{J}+80 \mathrm{~ms}$ and the $\mathrm{J}$ point was $\leq 0.5 \mathrm{~mm}$, the ST segment was classified as horizontal. If the measurement point $\mathrm{J}+80 \mathrm{~ms}$ was $>0.5 \mathrm{~mm}$ higher than the $\mathrm{J}$ point, the ST slope was labeled as positive (upsloping ST segment). Finally, if the measurement point $\mathrm{J}+80$ ms was $>0.5 \mathrm{~mm}$ lower than the $\mathrm{J}$ point, the ST slope was classified as negative (downsloping ST segment). The lead with the lowest single measurement at J point or J $+80 \mathrm{~ms}$ was selected to represent the lead group. Fig. 1 shows an example of a downsloping ST segment, and also the measurement points: the J point and $\mathrm{J}+80 \mathrm{~ms}$.

\section{Follow-up}

The data for mortality and follow-up until the end of the year 2015 were gathered from the Causes of Death register maintained by Statistics Finland. It contains 100\% of deaths of Finnish citizens in Finland and almost 100\% abroad. Databases were linked using a personal identity code. The endpoint of this study was mortality from any cause and the follow-up time was the time between entering the study and time of death or the end of follow up at the end of 2015.

\section{Medication}

Information about the prescribed baseline medication was gathered by checking the study participants' personal health insurance cards for rights of drug reimbursements and interviewing the study participants about prescription and non-prescription medicines. The names of the medications were checked from prescriptions or packages. Also, data from a separate registry (Statistics on reimbursements for prescription medicines: The Social Insurance Institution of Finland) was included in the definition of coronary heart disease (CHD).

\section{Definitions of CHD, myocardial infarction, LVH and chronic obstructive pul- monary disease}

Trained study personnel performed the health interview, and they followed a structural detailed written instruction to gather information about pre-existent diseases. Examining physicians performed another structured interview and physical examination. Information on CHD was obtained from the Care Register for Health Care maintained by National Institute for Health and Welfare, which contains data of all

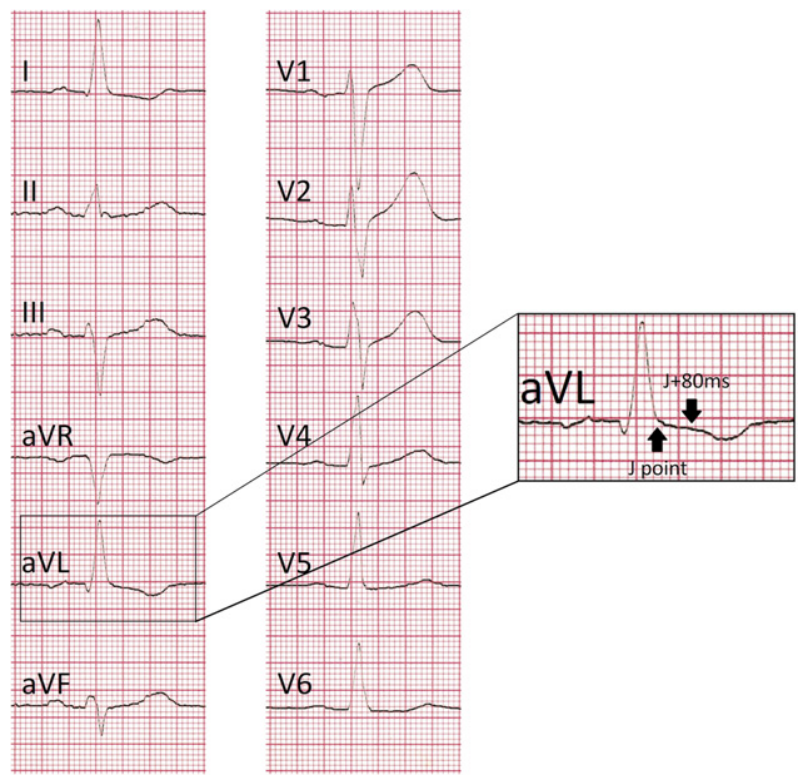

Fig. 1. An ECG example of a 59-year-old male participant who died during the follow-up. The ST segment in lead aVL is downsloping. Compared with the reference line, the level of the J point is $-0.15 \mathrm{~mm}$, while the level at the measurement point $\mathrm{J}+80 \mathrm{~ms}$ is $-0.75 \mathrm{~mm}$. The lowest J point in the lateral leads is in V5 $(-0.40 \mathrm{~mm})$. The ECG was recorded at $50 \mathrm{~mm} / \mathrm{s}$ and $10 \mathrm{~mm} / \mathrm{mV}$. 
inpatient episodes in Finland at the individual level. The accuracy of the registers has been validated previously $[15,16]$.

Classification of CHD required at least one of the following: diagnosed angina pectoris, myocardial infarction, percutaneous coronary intervention (PCI) or bypass surgery by examining physician, diagnosed PCI or bypass surgery in the health interview, ICD-codes 410-414 (ICD8/9) or I20-I25 (ICD10) in the Care Register for Health Care before the reference date of the study, or the right for drug reimbursements for CHD. Classification of myocardial infarction required either a diagnosis of a history of a myocardial infarction by the examining physician, large Q waves in the resting ECG or a history of myocardial infarction in the Care Register for Health Care, ICD-codes 410 (ICD8/9) or I21-I22 (ICD10). LVH was defined by Minnesota code criteria 3-1, 3-3 or 3-4 [17]. For the diagnosis of chronic obstructive pulmonary disease (COPD), information gathered during the health interview was used.

\section{Other measurements and definitions}

Height, weight and waist circumference were measured and body mass index (BMI) was calculated. Blood pressure was measured from the right arm with a standard mercury manometer (Mercuro 300; Speidel \& Keller, Jungingen, Germany). An average of two measurements was used, of which the first was measured after rest for at least 5 min in sitting position. Hypertension (arterial hypertension, HTA) was defined as a blood pressure $\geq 140 / 90$ at the baseline.

Serum total cholesterol, high-density lipoprotein cholesterol (HDL), low-density lipoprotein cholesterol (LDL), triglyceride and plasma glucose concentrations were determined enzymatically from venous blood samples with a clinical chemistry analyzer (Olympus, AU400, Hamburg, Germany). The diagnosis of diabetes mellitus (DM) at baseline was determined as fasting serum glucose (fS-Gluc) $\geq 7$ or a history of use of oral glucose lowering agents or insulin injections [18].

\section{Exclusion criteria}

We excluded subjects with missing ECG data $(n=55)$. Of those, the recording was unsuccessful in 36 participants with entries such as "difficult to move," "wheelchair", "denial”, "leg/hand amputated", "in geriatric chair", "massive hernia", "plaster in leg/hand". In the further process, 19 ECGs were lost (diskette lost 9, coupling error 4, data reading failure 5 , unspecific reason 1 ). We also excluded subjects with either large Q/QS waves in the ECG using Minnesota codes 1-1, 1-2 and 1-3 $(\mathrm{n}=127)$, left or right bundle branch block or left anterior hemiblock (Minnesota code 7, $\mathrm{n}=565$ ), Wolf-Parkinson-White pattern (Minnesota code $6-4, n=1$ ) or paced rhythm (Minnesota code $6-8, n=4$ ) from the analysis. Thereby, the final study population consisted of 5613 subjects, of which 2462 were males and 3151 females.

\section{Statistical analysis}

All analyses were done separately for men and women. Comparisons in variables were calculated with either $t$-test, Mann-Whitney U or Chisquare tests as appropriate. ST levels were treated as continuous variables. The normality of the distribution of ST-segment levels was estimated with Q-Q plots and histograms. The linearity of the association of ST level and mortality was checked in a spline model. After visual inspection the association did not significantly differ from linear. Hence, we did not test the linearity in other ways. Cox proportional hazard models were constructed separately for minimum ST levels and ST slopes in every lead group and at all measurement points. Total mortality was used as the endpoint. Regarding ST slope, the three previously mentioned categories (upsloping, horizontal, downsloping) were used and the upsloping ST segment was used as reference. For ST levels, hazard ratios (HR) were scaled for a change of $1.0 \mathrm{~mm}$. The following parameters were used for multivariate adjusting: age, BMI, HDL, LDL, HTA, DM and CHD. Another model was also constructed, where known CHD was excluded from the multivariate adjustment. The linearity analyses were done using $\mathrm{R}$ and all other analyses were performed with SPSS 25. Statistical significance was based on $\mathrm{p}<0.05$.

\section{Results}

Table 1 shows the baseline characteristics and medication of the study population. Compared with survivors, the non-survivors were older and more often had co-morbidities. There was higher proportion of subjects with ECG-LVH in the female non-survivor group compared to the survivor group, while there was no statistically significant difference in male subjects in a similar comparison. The use of all studied medication groups was clearly more frequent among non-survivors than among survivors. Table 2 shows the mean ST levels and the type of ST slope in the three lead groups and lead V5 separately for women and men and for survivors and non-survivors. Non-survivors had relatively lower ST levels in both men and women in every lead group, and women tended to have slightly lower ST levels than men. The variation of the ST levels was quite low as the maximum standard deviation (SD) was $0.54 \mathrm{~mm}$. Non-survivors also had a higher proportion of negative ST slopes, especially in women. However, negative ST slope was a quite rare finding with a prevalence of $0.1 \%$ to $5.4 \%$ in the population in the different lead groups.

The mean follow-up time was 13.7 years (SD 3.3 years) for men and 13.9 years for women (SD 3.1 years). Of the male subjects, $487(19.8 \%)$ died during follow-up compared to 555 (17.6\%) women.

\section{ST level and outcome}

Lower ST levels were associated with worse outcome in every lead group at all measurement points and in both genders. Adverse outcome was most evident in the lateral lead group at $\mathrm{J}+80 \mathrm{~ms}$ with a $\mathrm{HR}$ of 0.13 (95\% confidence interval $[\mathrm{CI}] 0.11-0.15, \mathrm{p}<0.001$ ) in women and $\mathrm{HR}$ 0.21 (CI $0.17-0.25, \mathrm{p}<0.001$ ) in men for a change of $1.0 \mathrm{~mm}$. Fig. 2 shows the linearity of the unadjusted association of lateral ST levels and mortality at the measurement point $J_{\text {alt }} \mathrm{J}+80 \mathrm{~ms}$. After adjustment for age, the increase in risk of lower ST levels was diminished in the inferior leads, and also for the anterior leads in men. Lower ST level in the lateral leads at all measurement points in both genders retained its statistical significance for increased mortality after adjusting with age. This was also the case in women at the measurement point $\mathrm{J}+80 \mathrm{~ms}$ and for $\mathrm{Jalt}_{\mathrm{al}}+80 \mathrm{~ms}$ in the anterior leads and V5. For all the other measurement points and lead groups, the statistical significance was lost after adjustment for age. After multivariate adjustment, lower ST levels were associated with higher mortality rates in the lateral leads in both genders at all measurement points and in $\mathrm{V} 5$ at $\mathrm{J}_{\mathrm{alt}} \mathrm{J}+80 \mathrm{~ms}$ in men. At all the other measurement points and lead groups, the statistical significance was lost after multivariate adjustment. The multi-adjusted hazard ratios are shown in Table 3.

\section{Significance of ECG-LVH and associated CHD}

In multi-adjusted analysis, after the exclusion of the subjects with ECG-LVH, the mortality risk of lower ST levels in the lateral leads increased in men. The same was true for V5 both at the J point and $\mathrm{J}_{\mathrm{alt}} \mathrm{J}+80$ ms: HR 0.69 (CI 0.51-0.92, p = 0.011) and HR $0.70(\mathrm{Cl}$ $0.52-0.94, p=0.019$ ), respectively. However, the effect of excluding ECG-LVH patients was opposite in women, where only the measurement point $\mathrm{J}+80 \mathrm{~ms}$ retained its statistical significance in the lateral leads. Fig. 3 shows the HRs in the multi-adjusted model in lateral leads separately for men and women after exclusion of subjects with ECG-LVH. On the other hand, excluding subjects with CHD from multivariate adjustment had no clear influence on the results: lower lateral ST levels remained a significant risk factor in both genders (Fig. 3). There was one exception: in lead V5 in women, ST level at J $+80 \mathrm{~ms}$ proved to be associated with worse outcome (HR 0.81 [CI 0.67-0.98, 
Table 1

Baseline characteristics of the Health 2000 Survey participants.

\begin{tabular}{|c|c|c|c|c|c|c|c|c|c|c|}
\hline \multirow{3}{*}{ Variable } & \multicolumn{5}{|c|}{ Men } & \multicolumn{5}{|c|}{ Women } \\
\hline & \multicolumn{2}{|c|}{ Survivors } & \multicolumn{2}{|c|}{ Non-survivors } & \multirow{2}{*}{$\begin{array}{l}\mathrm{p} \\
\text { value }\end{array}$} & \multicolumn{2}{|c|}{ Survivors } & \multicolumn{2}{|c|}{ Non-survivors } & \multirow{2}{*}{$\begin{array}{l}\mathrm{p} \\
\text { value }\end{array}$} \\
\hline & Mean/Median/N & $\mathrm{SD} / \mathrm{Q}_{1}-\mathrm{Q}_{3} /(\%)$ & Mean/Median/N & $\mathrm{SD} / \mathrm{Q}_{1}-\mathrm{Q}_{3} /(\%)$ & & Mean/Median/N & $\mathrm{SD} / \mathrm{Q}_{1}-\mathrm{Q}_{3} /(\%)$ & Mean/Median/N & $\mathrm{SD} / \mathrm{Q}_{1}-\mathrm{Q}_{3} /(\%)$ & \\
\hline $\mathrm{N}$ & 1975 & $(80.2 \%)$ & 487 & $(19.8 \%)$ & & 2596 & $(82.4 \%)$ & 555 & $(17.6 \%)$ & \\
\hline Age & 47.2 & 10.9 & 64.4 & 13.5 & $<0.001$ & 48.7 & 12.0 & 72.6 & 12.2 & $<0.001$ \\
\hline BMI $\left(\mathrm{kg} / \mathrm{m}^{2}\right)$ & 27.1 & 4.0 & 27.1 & 4.5 & 0.773 & 26.5 & 5.0 & 28.0 & 5.0 & $<0.001$ \\
\hline $\begin{array}{l}\text { Waist circumference } \\
(\mathrm{cm})\end{array}$ & 97.3 & 11.1 & 99.3 & 12.3 & 0.002 & 87.3 & 13.1 & 93.2 & 12.6 & $<0.001$ \\
\hline Heart rate/min & 61.7 & 10.7 & 65.7 & 12.9 & $<0.001$ & 63.6 & 10.2 & 66.9 & 11.8 & $<0.001$ \\
\hline Regular smoking & 533 & $(27.1 \%)$ & 150 & $(30.9 \%)$ & 0.092 & 472 & $(18.3 \%)$ & 59 & $(10.7 \%)$ & $<0.001$ \\
\hline COPD & 18 & $(0.9 \%)$ & 19 & $(3.9 \%)$ & $<0.001$ & 19 & $(0.7 \%)$ & 18 & $(3.2 \%)$ & $<0.001$ \\
\hline Hypertension & 832 & $(42.2 \%)$ & 297 & $(61.0 \%)$ & $<0.001$ & 828 & $(32.0 \%)$ & 367 & $(66.5 \%)$ & $<0.001$ \\
\hline Diabetes & 103 & $(5.2 \%)$ & 69 & $(14.2 \%)$ & $<0.001$ & 79 & $(3.0 \%)$ & 81 & $(14.6 \%)$ & $<0.001$ \\
\hline LVH & 419 & $(21.2 \%)$ & 95 & (19.5\%) & 0.406 & 201 & $(7.7 \%)$ & 105 & $(18.9 \%)$ & $<0.001$ \\
\hline CHD & 76 & $(3.8 \%)$ & 95 & $(19.5 \%)$ & $<0.001$ & 73 & $(2.8 \%)$ & 132 & $(23.8 \%)$ & $<0.001$ \\
\hline $\begin{array}{l}\text { Myocardial } \\
\text { infarction }\end{array}$ & 21 & $(1.1 \%)$ & 36 & $(7.4 \%)$ & $<0.001$ & 11 & $(0.4 \%)$ & 30 & $(5.4 \%)$ & $<0.001$ \\
\hline $\begin{array}{l}\text { Total cholesterol } \\
(\mathrm{mmol} / \mathrm{L})\end{array}$ & 6.0 & 1.1 & 5.9 & 1.2 & 0.056 & 5.8 & 1.1 & 6.2 & 1.2 & $<0.001$ \\
\hline $\mathrm{HDL}(\mathrm{mmol} / \mathrm{L})$ & 1.2 & 0.3 & 1.2 & 0.4 & 0.543 & 1.5 & 0.4 & 1.3 & 0.4 & $<0.001$ \\
\hline $\mathrm{LDL}(\mathrm{mmol} / \mathrm{L})$ & 3.9 & 1.0 & 3.7 & 1.1 & 0.002 & 3.6 & 1.0 & 3.9 & 1.1 & $<0.001$ \\
\hline $\begin{array}{l}\text { Triglycerides } \\
(\mathrm{mmol} / \mathrm{L})\end{array}$ & 1.5 & $1.1-2.2$ & 1.4 & $1.1-2.0$ & 0.478 & 1.2 & $0.9-1.6$ & 1.5 & $1.1-2.0$ & $<0.001$ \\
\hline CRP (mg/L) & 0.7 & $0.33-1.6$ & 1.2 & $0.4-3.1$ & $<0.001$ & 0.7 & $0.2-1.9$ & 1.1 & $0.4-3.1$ & $<0.001$ \\
\hline Uric acid $(\mu \mathrm{mol} / \mathrm{L})$ & 337.5 & 69.0 & 348.9 & 81.7 & 0.005 & 258.2 & 65.7 & 310.0 & 86.3 & $<0.001$ \\
\hline \multicolumn{11}{|l|}{ Medication } \\
\hline Beta-blocker & 158 & $(8.0 \%)$ & 116 & $(23.8 \%)$ & $<0.001$ & 300 & $(11.6 \%)$ & 172 & $(31.0 \%)$ & $<0.001$ \\
\hline $\mathrm{CCB}$ & 71 & $(3.6 \%)$ & 54 & $(11.1 \%)$ & $<0.001$ & 109 & $(4.2 \%)$ & 87 & $(15.7 \%)$ & $<0.001$ \\
\hline Digitalis & 9 & $(0.5 \%)$ & 19 & $(3.9 \%)$ & $<0.001$ & 8 & $(0.3 \%)$ & 43 & $(7.7 \%)$ & $<0.001$ \\
\hline ACEI/ARB & 124 & $(6.3 \%)$ & 72 & $(14.8 \%)$ & $<0.001$ & 155 & $(6.0 \%)$ & 92 & $(16.6 \%)$ & $<0.001$ \\
\hline
\end{tabular}

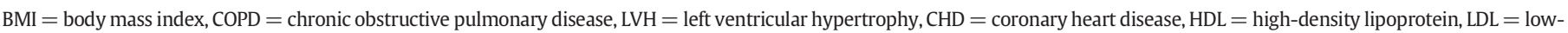

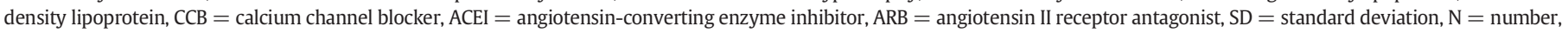
$\mathrm{Q}_{1}-\mathrm{Q}_{3}=$ quartiles.

$\mathrm{p}=0.033])$. Regarding the anterior and inferior lead groups, excluding ECG-LVH subjects or adjustment for associated CHD did not influence the prognostic significance of ST levels (data not shown).

\section{ST slope}

A downsloping ST segment in the lateral leads was associated with higher mortality rates in both genders. This was most evident in lead V5 in women; 19 out of 22 female study participants with a downsloping ST segment died during the follow-up (HR 15.09 [CI 9.11-24.99, $\mathrm{p}<0.001])$. The corresponding HR for men was 3.69 (CI $1.37-9.92, \mathrm{p}=0.010$ ). In women, a negative ST slope was also associated with increased mortality in the anterior leads, while a horizontal ST, compared with upsloping ST, was associated with better outcome when present in the inferior leads ( $\mathrm{HR} 0.44$ [CI 0.32-0.62, p < 0.001]) and worse outcome when present in V5 (HR 1.51 [CI 1.19-1.92, p = $0.001]$ ). Also, in men, a horizontal slope in V5 was associated with worse outcome (HR 2.11 [CI 1.76-2.52, p < 0.001]). However, after adjusting for age, a downsloping ST segment did not affect outcome in men in any lead groups. In women, a downsloping or horizontal slope was associated with worse outcome in the lateral leads as did also a descending slope in V5. After multivariate adjustment, ST slope lost its statistical significance to predict mortality in all lead groups in both genders (data not shown). Excluding subjects with ECG-LVH did not have any significant effect on the multivariate adjusted results. However, a downsloping ST segment in the lateral leads proved to be a risk marker for all-cause mortality in women, when CHD was excluded from the multivariate adjustment model (HR 1.70 [CI 1.04-2.78, p = 0.036 ] compared to HR 1.62 [CI 0.99-2.66, $\mathrm{p}=0.056$ ] in multivariate adjusted model with $\mathrm{CHD}$ ).

Table 2

Mean ST (the lowest of J and J $+80 \mathrm{~ms}\left(\mathrm{Jalt}_{\mathrm{l}} \mathrm{H}+80 \mathrm{~ms}\right)$ ) levels and standard deviation (SD) and the percentages of different ST slope groups.

\begin{tabular}{|c|c|c|c|c|c|c|}
\hline \multirow{2}{*}{ Variable } & \multicolumn{3}{|c|}{ Men } & \multicolumn{3}{|c|}{ Women } \\
\hline & Survivors $(\mathrm{N}=1975)$ & Non-survivors $(\mathrm{N}=487)$ & $\mathrm{p}$ value & Survivors $(\mathrm{N}=2596)$ & Non-survivors $(\mathrm{N}=555)$ & $\mathrm{p}$ value \\
\hline \multicolumn{7}{|c|}{ ST segment, mean (mm) (SD) } \\
\hline Anterior & $0.01(0.54)$ & $-0.10(0.43)$ & $<0.001$ & $-0.13(0.27)$ & $-0.29(0.40)$ & $<0.001$ \\
\hline Lateral & $-0.04(0.26)$ & $-0.20(0.32)$ & $<0.001$ & $-0.20(0.32)$ & $-0.32(0.38)$ & $<0.001$ \\
\hline Inferior & $-0.11(0.34)$ & $-0.16(0.28)$ & $<0.001$ & $-0.09(0.24)$ & $-0.16(0.32)$ & $<0.001$ \\
\hline V5 & $0.20(0.38)$ & $-0.05(0.42)$ & $<0.001$ & $0.01(0.27)$ & $-0.22(0.43)$ & $<0.001$ \\
\hline \multicolumn{7}{|c|}{ ST slope, percentages (positive/horizontal/negative) } \\
\hline Anterior & $58.9 / 40.9 / 0.2$ & $60.4 / 40.6 / 0.2$ & 0.846 & $44.1 / 55.8 / 0.1$ & $46.7 / 52.6 / 0.7$ & 0.003 \\
\hline Lateral & $21.3 / 72.2 / 0.5$ & $19.9 / 78.4 / 1.6$ & 0.026 & $8.9 / 90.4 / 0.7$ & $6.8 / 87.7 / 5.4$ & $<0.001$ \\
\hline Inferior & $4.1 / 93.1 / 2.8$ & $6.2 / 92.0 / 1.8$ & 0.080 & $3.0 / 96.0 / 1.0$ & $7.0 / 91.0 / 2.0$ & $<0.001$ \\
\hline V5 & $63.6 / 36.1 / 0.3$ & 43.3/55.9/0.8 & $<0.001$ & $20.7 / 79.2 / 0.1$ & $13.9 / 82.7 / 3.4$ & $<0.001$ \\
\hline
\end{tabular}

$\mathrm{N}=$ number, $\mathrm{SD}=$ standard deviation. 

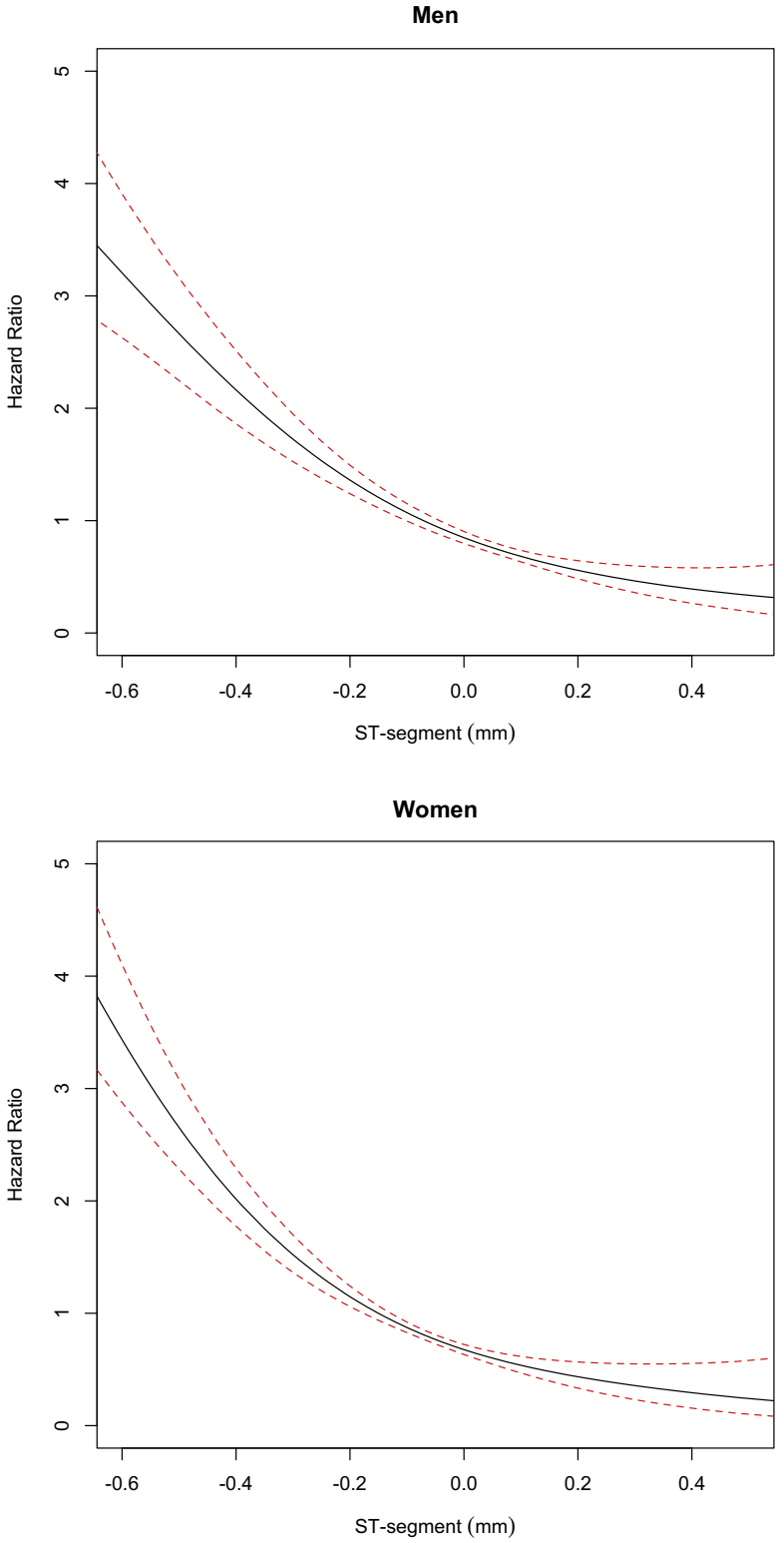

Fig. 2. The unadjusted hazard ratios between ST segment and mortality at measurement point $\mathrm{J}_{\text {alt }} \mathrm{J}+80 \mathrm{~ms}$ (lower of J point and $\mathrm{J}+80 \mathrm{~ms}$ ) in lateral leads in men and in women among normal ranges of ST-level measurements. The risk for mortality increases along the lower ST levels.

\section{Discussion}

Our population study, with a follow-up of nearly 14 years, showed that lower ST level in the lateral ECG leads (I, aVL, V5, V6) as a continuous variable is independently associated with all-cause mortality in both women and men. ST changes in the lateral leads had clearly negative prognostic impact, while anterior or inferior ST level had no prognostic significance after multivariate adjustment. Some previous studies have also reported consistency of prognosis between the genders especially regarding minor, non-specific or "borderline" ST changes in the general population $[3,4,12,13]$. We found that age has strong impact on the ST level, as was also documented previously [4,5].

All analyses were done separately for men and women because of the previously described gender differences related to the relative ST segment and the T wave in the 12-lead ECG [11]. We also wanted to study gender-specific differences in different lead groups and measurement points. In previous studies, the prevalence of minor ST changes has been greater in women than in men [4]. Also in our study, women
Table 3

Mortality of continuous ST levels in different lead groups in men and women, multivariate adjusted $^{\mathrm{a}}$ Cox regression analysis. Hazard ratios are scaled for a change of $1.0 \mathrm{~mm}$.

\begin{tabular}{|c|c|c|c|c|}
\hline & \multicolumn{2}{|l|}{ Men } & \multicolumn{2}{|l|}{ Women } \\
\hline & Hazard ratio $(95 \% \mathrm{CI})$ & $\mathrm{p}$ value & Hazard ratio $(95 \% \mathrm{CI})$ & $\mathrm{p}$ value \\
\hline \multicolumn{5}{|l|}{ Anterior } \\
\hline J point & $1.06(0.85-1.30)$ & 0.621 & $0.93(0.72-1.20)$ & 0.557 \\
\hline $\mathrm{J}+80 \mathrm{~ms}$ & $1.11(0.90-1.36)$ & 0.318 & $0.85(0.68-1.05)$ & 0.134 \\
\hline $\mathrm{Jalt}_{\mathrm{J}} \mathrm{J}+80 \mathrm{~ms}$ & $1.05(0.85-1.30)$ & 0.641 & $0.90(0.70-1.15)$ & 0.411 \\
\hline \multicolumn{5}{|c|}{ Lateral } \\
\hline J point & $0.70(0.54-0.91)$ & 0.008 & $0.70(0.53-0.91)$ & 0.009 \\
\hline $\mathrm{J}+80 \mathrm{~ms}$ & $0.64(0.49-0.84)$ & 0.002 & $0.61(0.48-0.78)$ & $<0.001$ \\
\hline $\mathrm{J}_{\mathrm{alt}} \mathrm{J}+80 \mathrm{~ms}$ & $0.69(0.53-0.89)$ & 0.005 & $0.68(0.54-0.87)$ & 0.002 \\
\hline \multicolumn{5}{|c|}{ Inferior } \\
\hline J point & $1.12(0.85-1.48)$ & 0.433 & $0.96(0.72-1.29)$ & 0.781 \\
\hline $\mathrm{J}+80 \mathrm{~ms}$ & $1.09(0.78-1.52)$ & 0.611 & $0.93(0.68-1.26)$ & 0.625 \\
\hline $\mathrm{Jalt}_{\mathrm{a}} \mathrm{J}+80 \mathrm{~ms}$ & $1.16(0.86-1.57)$ & 0.323 & $0.93(0.69-1.26)$ & 0.639 \\
\hline \multicolumn{5}{|c|}{ V5 } \\
\hline J point & $0.80(0.63-1.01)$ & 0.057 & $0.85(0.67-1.08)$ & 0.190 \\
\hline $\mathrm{J}+80 \mathrm{~ms}$ & $0.85(0.71-1.03)$ & 0.094 & $0.84(0.69-1.03)$ & 0.086 \\
\hline $\mathrm{Jalt}_{\mathrm{J}} \mathrm{J}+80 \mathrm{~ms}$ & $0.79(0.63-1.00)$ & 0.046 & $0.83(0.67-1.05)$ & 0.116 \\
\hline
\end{tabular}

had lower mean ST levels than men. In the anterior leads, age seemed to explain the association between lower ST levels and mortality in men, but in women, lower anterior ST levels increased the risk for mortality also after adjusting with age. However, the HRs did not differ significantly between sexes after multivariate adjustment.

ECG-LVH produced strikingly different prognostic implications between the genders. In women after excluding subjects with ECG-LVH, only the J +80 ms measurement point retained its power as a negative prognostic marker for all-cause death after multivariate adjustment. For the male subjects, excluding those with ECG-LVH tended to strengthen the effect of lower lateral ST levels on mortality. Previously, when we studied continuous ST depression and cardiovascular mortality in the same population with shorter follow-up, we found a similar effect of the exclusion of ECG-LVH patients in individuals older than 55 years [11]. The results of our study indicate that the prognostic significance of a lower lateral ST level at the J point in women is, at least to some extent, explained by ECG-LVH. In men, including ECG-LVH into the analyses had an opposite effect, indicating that the adverse prognostic effect of this ECG change is not related to ECG-LVH. We have no definite explanation for this gender difference. In our population, there was a higher proportion of subjects with ECG-LVH in the female non-survivor group compared to the survivor group, while there was no statistically significant difference in men (Table 1). Also, more male than female study participants were excluded from the statistical analyses when studying the effect of ECG-LVH. Considering the large number of study participants, we think that this difference did not have major impact on the results, although this possibility cannot be excluded with certainty. In an earlier population study of Larsen et al. [1], the prevalence of voltage-only ECG-LVH increased with age in women but decreased in men, which could be one possible explanation for the observed gender difference.

Many previous studies have evaluated the significance of ST depression after excluding subjects with known CHD. Interestingly, adjusting for CHD did not have any major influence on our results. The definition of CHD in our study included many alternative (even though relevant) criteria, and the vast majority of subjects with a CHD diagnosis is likely to be covered. Participants with asymptomatic, and thus undiagnosed CHD, could dilute the results. However, it should be kept in mind that the ST level is not normally affected by stable CHD. In a study by Walsh et al., minor ST-segment and T-wave abnormalities were not associated with the presence of coronary artery calcium in participants free of clinical CHD at baseline [19]. Our study results indicate that 
Men

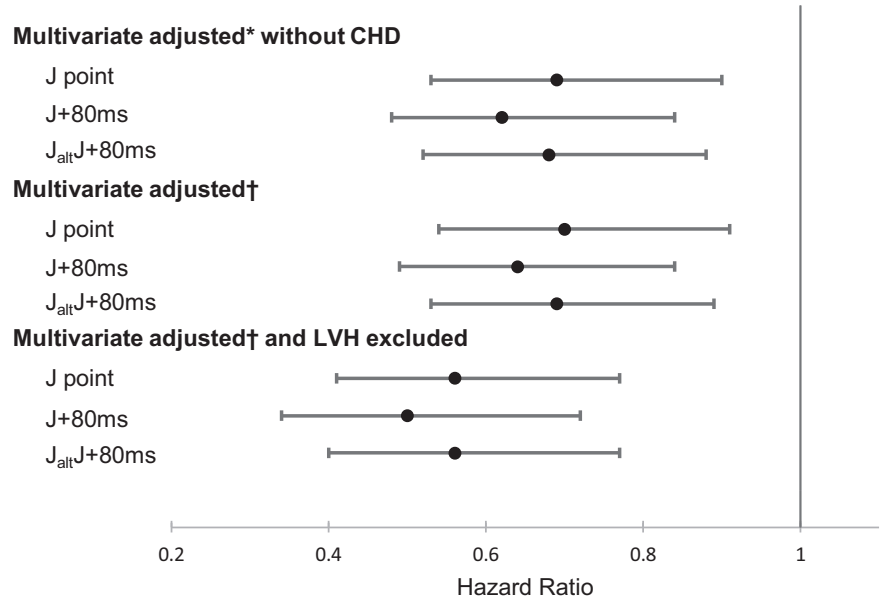

\section{Women}

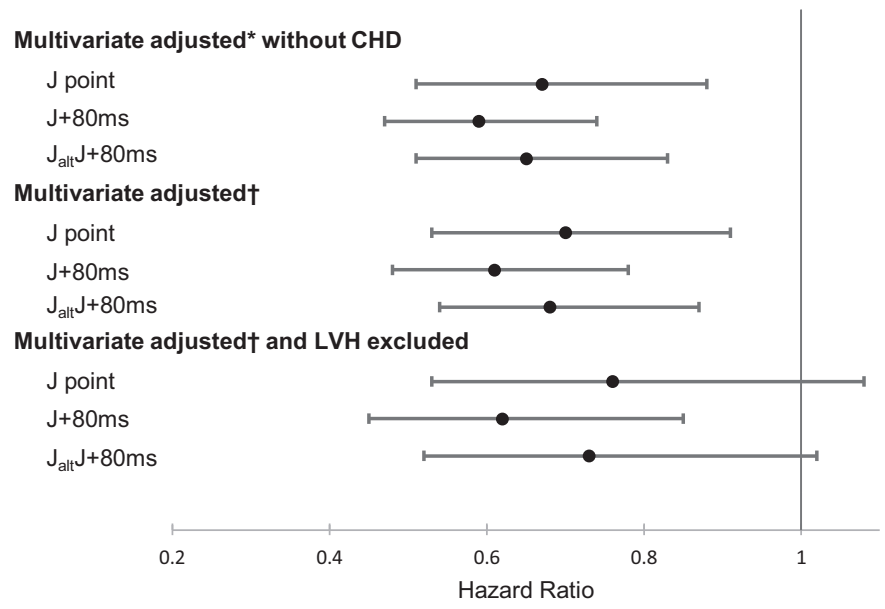

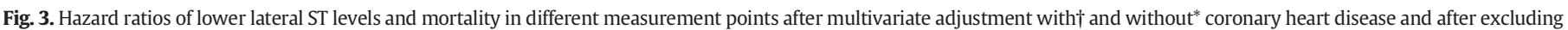

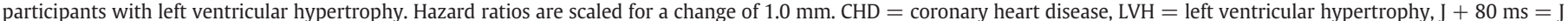

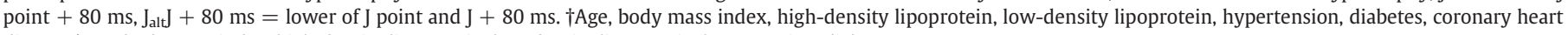
disease. *Age, body mass index, high-density lipoprotein, low-density lipoprotein, hypertension, diabetes.

other etiologies may be more important than CHD to explain the prognostic role on changes in the ST level in the lateral ECG leads. In some studies, minor ST segment changes associated with cardiovascular mortality, but not with non-fatal myocardial infarction, indicating that arrhythmias could be one of the causes of the association [20].

Sympathetic activity is known to cause ST depression in healthy subjects, and it is a known risk factor for cardiac death and arrhythmias $[21,22]$. The use of digoxin, which clearly may affect the ST level and ST slope, was quite rare in this study. In structural heart disease, ECGLVH often accompanies ST depression. In the present study, the negative prognostic impact of low lateral ST level remained after the exclusion of ECG-LVH in men and also in women at $\mathrm{J}+80 \mathrm{~ms}$. Structural heart disease often results in ventricular conduction defects with broad QRS, but this was an exclusion criterion in this study.

\section{The prognostic role of the ST slope}

The early repolarization pattern with a horizontal ST segment has been associated with cardiovascular and all-cause mortality, while an elevated J point with upsloping ST segment has not been associated with adverse prognosis in population studies $[23,24]$. The "strain pattern", ST depression with downsloping ST and T-wave inversion, is a well-recognized marker of anatomical LVH and is associated with larger left ventricular mass and worse outcome in HTA [25,26], and was associated with all-cause mortality, systolic dysfunction, and myocardial scar in multiethnic participants of a population study [10]. To our knowledge, the role of the ST-segment morphology independently of the level of the ST segment has not been established before.

We found that a downsloping ST segment was associated with increased all-cause mortality in the lateral leads (and V5) in both genders and in the anterior leads in women regardless of the level of the ST segment. Interestingly, in our study, a horizontal ST segment in the inferior leads was even associated with a better prognosis than an upsloping ST in women. In V5, the associated mortality increased among the groups in both genders: a horizontal ST segment was associated with higher mortality rates compared to an upsloping ST segment, and a downsloping ST segment was associated with even higher mortality rates. After multivariate adjustment, a downsloping ST segment lost its significance in all lead groups in both genders, but in the multiadjusted model without CHD, lateral negative slope increased mortality in women. We hypothesize that CHD should be suspected in case of a downsloping ST segment in the lateral leads in women. 
Lead groups and measurement points

Most of the previous population studies classified ST changes based on the Minnesota coding, ignoring the location of ST and T-wave changes within the 12-lead ECG. A few studies showed that the prognosis of ST changes vary depending on their location over different lead groups $[8,11]$. We divided the leads into three anatomical groups, and these carried clearly different prognostic values. Age seemed to explain the adverse prognosis of lower ST levels in the inferior and anterior leads, while lower lateral ST level remained as an independent risk factor for increased mortality also after multivariate adjustment.

The chosen measurement point for ST deviations vary between studies. Our earlier account from the same data with shorter follow-up [11] used three different measurement points. Neither shorter nor the current longer follow-up differentiated HRs between different measurement points. However, the increase in the risk was somewhat more pronounced when using the measurement point $\mathrm{J}+80$ both in multiadjusted models and after excluding ECG-LVH.

The variation of the ST level was small, and we used computerized measurements to measure the level of the ST segment. Automatic measurements help to correct for disturbances caused by wandering baseline and improve comparison between different ECGs. In our study HRs were scaled for difference of $1 \mathrm{~mm}$. However, our data suggest that smaller changes have prognostic effect. Computerized measurement of the ST level is necessary to perform reliable measurements in case of minor changes.

\section{Study limitations and strengths}

The Health 2000 population is a representative sample of the Finnish population 30 years of age or older. The results may not be applicable to other populations. The long follow-up time up to 15 years resulted in a relatively high mortality rate ( $19.8 \%$ in men and $17.6 \%$ in women) for a population study. As our study contained a relatively high number of statistical comparisons, borderline significances should be evaluated with caution. Absence of echocardiographic and magnetic resonance imaging data on LVH is a study limitation typical of a population study.

Continuous instead of categorical variable enhances sensitivity to detect subtle differences. Inclusion of lead groups for ST analyses is important, because the prognostic information clearly differs between the different categories. The lead with the lowest ST level was chosen to represent a lead group. This makes our results easy to implement into clinical practice.

We examined ST slope independently of the level of ST segment. It is possible that the combination of slope with ST depression would have altered the study results. This was not the aim of our study, because the prognostic significance of the lateral "strain pattern" has already been established in the general population, in hypertension and structural heart disease.

\section{Conclusion}

Lower levels of the ST segment in the lateral lead group as a continuous variable is associated with increased mortality in the general population independently of gender, other cardiovascular risk factors and CHD. The prognosis is highly dependent on the lead-related spatial region: in the anterior and inferior lead groups, the ST level did not affect prognosis after multivariate adjustment. In women, the prognostic effect of a lower lateral ST level, measured at the J point, is likely explained by ECG-LVH. Regarding the measurement point $\mathrm{J}+80 \mathrm{~ms}$ and in male individuals, more studies are required to understand the pathophysiology of the phenomenon. A downward slope of the ST segment in the lateral leads was associated with all-cause mortality, but the increased risk seemed to be explained by age and associated CHD.

\section{CRediT authorship contribution statement}

Tiia Istolahti: Conceptualization, Methodology, Formal analysis, Writing - original draft. Tuomo Nieminen: Conceptualization, Methodology, Writing - review \& editing. Heini Huhtala: Methodology, Formal analysis. Leo-Pekka Lyytikäinen: Software, Writing - review \& editing. Mika Kähönen: Supervision, Writing - review \& editing. Terho Lehtimäki: Writing - review \& editing. Markku Eskola: Writing - review \& editing. Ismo Anttila: Writing - review \& editing. Antti Jula: Data curation, Writing - review \& editing. Harri Rissanen: Resources, Writing - review \& editing. Kjell Nikus: Conceptualization, Methodology, Writing - original draft, Supervision, Project administration. Jussi Hernesniemi: Methodology, Formal analysis, Writing - review \& editing, Supervision.

\section{Declaration of competing interest}

None.

\section{Acknowledgements}

We thank the investigators, staff and participants of the Health 2000 survey.

\section{Source of funding}

This work was supported by The Doctoral Programme in Medicine and Life Sciences, Faculty of Medicine and Health Technology, Tampere University; Finnish Medical Foundation; Finska Läkaresällskapet; Academy of Finland (grant 322098); Competitive State Research Financing of the Expert Responsibility area of Tampere; Finnish Foundation for Cardiovascular Research; Tampere Tuberculosis Foundation; EU Horizon 2020 (grant 755320 for TAXINOMISIS); and Tampere University Hospital Supporting Foundation.

\section{References}

[1] Larsen CT, Dahlin J, Blackburn H, Scharling H, Appleyard M, Sigurd B, et al. Prevalence and prognosis of electrocardiographic left ventricular hypertrophy, ST segment depression and negative T-wave. The Copenhagen City Heart Study. Eur Heart J 2002;23(4):315-24. https://doi.org/10.1053/euhj.2001.2774 Feb 1.

[2] Möller CS, Zethelius B, Sundström J, Lind L. Persistent ischaemic ECG abnormalities on repeated ECG examination have important prognostic value for cardiovascular disease beyond established risk factors: a population-based study in middle-aged men with up to 32 years of follow-up. Heart 2007;93(9):1104-10. https://doi.org/ 10.1136/hrt.2006.109116 Sep 1.

[3] Rautaharju PM, Ge S, Nelson JC, Marino Larsen EK, Psaty BM, Furberg CD, et al. Comparison of mortality risk for electrocardiographic abnormalities in men and women with and without coronary heart disease (from the Cardiovascular Health Study). Am J Cardiol 2006;97(3):309-15. https://doi.org/10.1016/j.amjcard.2005.08.046 Feb.

[4] Kumar A, Lloyd-jones DM. Clinical significance of minor nonspecific St-segment and T-wave abnormalities in asymptomatic subjects: a systematic review. Cardiol Rev 2007;15(3):133-42. https://doi.org/10.1097/01.crd.0000249382.65955.14 May 1.

[5] Badheka AO, Rathod A, Marzouka GR, Patel N, Bokhari SSI, Moscucci M, et al. Isolated nonspecific ST-segment and T-wave abnormalities in a cross-sectional United States population and mortality (from NHANES III). Am J Cardiol 2012;110(4):521-5. https://doi.org/10.1016/j.amjcard.2012.04.023 Aug.

[6] Greenland P, Xie X, Liu K, Colangelo L, Liao Y, Daviglus ML, et al. Impact of minor electrocardiographic ST-segment and/or T-wave abnormalities on cardiovascular mortality during long-term follow-up. Am J Cardiol 2003;91(9):1068-74. https:// doi.org/10.1016/s0002-9149(03)00150-4 May 1.

[7] Daviglus ML, Liao Y, Greenland P, Dyer AR, Liu K, Xie X, et al. Association of nonspecific minor ST-T abnormalities with cardiovascular mortality: the Chicago Western Electric Study. JAMA 1999;281(6):530-6. https://doi.org/10.1097/00132586200002000-00057 Feb 10.

[8] Rasmussen PV, Nielsen JB, Pietersen A, Graff C, Lind B, Struijk JJ, et al. Electrocardiographic precordial ST-segment deviations and the risk of cardiovascular death: results from the Copenhagen ECG study. J Am Heart Assoc Cardiovasc Cerebrovasc Dis 2014;3(3). https://doi.org/10.1161/jaha.113.000549 Internet. Available from: https://www.ncbi.nlm.nih.gov/pmc/articles/PMC4309043/. May 9 [cited 2018 Dec 23].

[9] Froelicher VF, Myers J. Exercise and the heart. vol. 2000. Pennsylvania, Philadelphia: W.B. Saunders Co. 
[10] Inoue YY, Soliman EZ, Yoneyama K, Ambale-Venkatesh B, Wu CO, Sparapani R, et al. Electrocardiographic strain pattern is associated with left ventricular concentric remodeling, scar, and mortality over 10 years: the multi-ethnic study of atherosclerosis. J Am Heart Assoc Cardiovasc Cerebrovasc Dis 2017:6(9). https://doi.org/10.1161/ jaha.117.006624 Internet. Available from: https://www.ncbi.nlm.nih.gov/pmc/articles/PMC5634304/. Sep 20 [cited 2019 Feb 9].

[11] Anttila I, Nikus K, Kähönen M, Jula A, Reunanen A, Salomaa V, et al. Prognostic implications of quantitative ST-segment characteristics and T-wave amplitude for cardiovascular mortality in a general population from the Health 2000 Survey. Ann Med 2010;42(7):502-11. https://doi.org/10.3109/07853890.2010.505932 Oct 1.

[12] De Bacquer D, De Backer G, Kornitzer M, Myny K, Doyen Z, Blackburn H. Prognostic value of ischemic electrocardiographic findings for cardiovascular mortality in men and women. J Am Coll Cardiol 1998;32(3):680-5. https://doi.org/10.1016/s07351097(98)00303-9 Sep 1.

[13] De Bacquer D, De Backer G, Kornitzer M, Blackburn H. Prognostic value of ECG findings for total, cardiovascular disease, and coronary heart disease death in men and women. Heart 1998;80(6):570-7. https://doi.org/10.1136/hrt.80.6.570 Dec.

[14] Heistaro S, editor. Methodology report: Health 2000 survey. Publications of the National Public Health Institute B26/; 2008. http://urn.fi/URN:NBN:fi-fe201204193320.

[15] Sund R. Quality of the Finnish Hospital Discharge Register: a systematic review. Scand J Public Health 2012;40(6):505-15. https://doi.org/10.1177/ 1403494812456637 Aug 1.

[16] Rapola JM, Virtamo J, Korhonen P, Haapakoski J, Hartman AM, Edwards BK, et al. Validity of diagnoses of major coronary events in national registers of hospital diagnoses and deaths in Finland. Eur J Epidemiol 1997;13(2):133-8 Feb 1.

[17] Prineas RJ, Crow RS, Blackburn HW. The Minnesota code manual of electrocardiographic findings. June Littleton, MA: John Wright-PSG, Inc; 1982.

[18] World Health Organization, International Diabetes Federation. Definition and diagnosis of diabetes mellitus and intermediate hyperglycaemia: report of a WHO/IDF consultation. Internet. [cited 2019 Apr 16]. Available from http://www.who.int/ diabetes/publications/diagnosis_diabetes2006/en/; 2006.
[19] Walsh JA, Prineas R, Soliman EZ, Liu K, Ning H, Daviglus ML, et al. Association of isolated minor non-specific ST-segment and T-wave abnormalities with subclinical atherosclerosis in a middle-aged, biracial population: Coronary Artery Risk Development in Young Adults (CARDIA) study. Eur J Prev Cardiol 2013;20(6):1035-41. https://doi.org/10.1177/2047487312460017 Dec 1.

[20] Kumar A, Prineas RJ, Arnold AM, Psaty BM, Furberg CD, Robbins J, et al. Prevalence, prognosis and implications of isolated minor non-specific ST-segment and T-wave abnormalities in older adults: Cardiovascular Health Study. Circulation 2008;118 (25):2790-6. https://doi.org/10.1161/circulationaha.108.772541 Dec 16.

[21] Toivonen L, Helenius K, Viitasalo M. Electrocardiographic repolarization during stress from awakening on alarm call. J Am Coll Cardiol 1997;30(3):774-9. https:// doi.org/10.1016/s0735-1097(97)00222-2 Jul.

[22] Quyyumi AA, Wright C, Fox K. Ambulatory electrocardiographic ST segment changes in healthy volunteers. Br Heart J 1983;50(5):460-4. https://doi.org/10.1136/hrt.50. 5.460 Nov.

[23] Adler A, Rosso R, Viskin D, Halkin A, Viskin S. What do we know about the "Malignant Form" of early repolarization? J Am Coll Cardiol 2013;62(10):863-8. https://doi.org/10.1016/j.jacc.2013.05.054 Sep.

[24] Rollin A, Maury P, Bongard V, Sacher F, Delay M, Duparc A, et al. Prevalence, prognosis, and identification of the malignant form of early repolarization pattern in a population-based study. Am J Cardiol 2012;110(9):1302-8. https://doi.org/10. 1016/j.amjcard.2012.06.033 Nov.

[25] Okin PM, Devereux RB, Nieminen MS, Jern S, Oikarinen L, Viitasalo M, et al. Relationship of the electrocardiographic strain pattern to left ventricular structure and function in hypertensive patients: the LIFE study. J Am Coll Cardiol 2001;38(2):514-20. https://doi.org/10.1016/s1062-1458(01)00566-9 Aug.

[26] Okin PM, Oikarinen L, Viitasalo M, Toivonen L, Kjeldsen SE, Nieminen MS, et al. Prognostic value of changes in the electrocardiographic strain pattern during antihypertensive treatment: the losartan intervention for end-point reduction in hypertension study (LIFE). Circulation 2009;119(14):1883-91. https://doi.org/10. 1161/circulationaha.108.812313 Apr 14. 